\title{
AUTOMATIC TOPOLOGY DERIVATION FROM IFC BUILDING MODEL FOR IN-DOOR INTELLIGENT NAVIGATION
}

\author{
S. J. Tang ${ }^{\text {b }}$, Q. Zhu ${ }^{\text {bc }}$, W. W. WANG ${ }^{\text {a }}$, Y.T. ZHANG ${ }^{\text {b }}$
}

\author{
${ }^{a}$ Shenzhen research center of digital city engineering, Shenzhen, P.R. China-measurer@163.com \\ ${ }^{\mathrm{b}}$ State Key Laboratory of Information Engineering in Surveying Mapping and \\ Remote Sensing, Wuhan University, Wuhan, P.R. China-shengjun.tang@whu.edu.cn \\ ${ }^{c}$ Faculty of Geosciences and Environmental Engineering of \\ Southwest Jiaotong University, Chengdu, P.R. China- zhuq66@263.net
}

Commission/WG

KEY WORDS: IFC, Geometry-Semantics-based, Topology Derivation, Navigation

\begin{abstract}
:
With the goal to achieve an accuracy navigation within the building environment, it is critical to explore a feasible way for building the connectivity relationships among 3D geographical features called in-building topology network. Traditional topology construction approaches for indoor space always based on 2D maps or pure geometry model, which remained information insufficient problem. Especially, an intelligent navigation for different applications depends mainly on the precise geometry and semantics of the navigation network. The trouble caused by existed topology construction approaches can be smoothed by employing IFC building model which contains detailed semantic and geometric information. In this paper, we present a method which combined a straight media axis transformation algorithm (S-MAT) with IFC building model to reconstruct indoor geometric topology network. This derived topology aimed at facilitating the decision making for different in-building navigation. In this work, we describe a multi-step deviation process including semantic cleaning, walkable features extraction, Multi-Storey 2D Mapping and S-MAT implementation to automatically generate topography information from existing indoor building model data given in IFC.
\end{abstract}

\section{INTRODUCTION}

As the growing complexity of Indoor environment and an increasing number of disasters (compartment fires, terrorist attack, etc.), there has been a practical requirements on indoor navigation approach, which is still one of the most challenging topics for research. A successful navigation depends mainly on the accuracy geometry and semantics of network ( $\mathrm{Li}$ and $\mathrm{He}$, 2008). As these complex geometry information can be easily obtained in outside by different 3D data capturing technologies (Shi et al., 2004), most researchers focus on the outdoor navigation environment. On the contrary, the information related to indoor still remains limited (Isikdag et al., 2013). Many cases of the indoor navigation process based on 2D maps or pure geometry information, which are inadequate for $3 \mathrm{D}$ topology deviation (Lee and Kwan, 2005). A successful navigation depends on correct semantic and structural information about building elements.

Many researches imply that Building Information Model (BIMs) can serve as a valuable information source for facilitating indoor navigation (Chen and Huang; Isikdag et al., 2013; Li, 2012; Rueppel and Stuebbe, 2008). A BIM model is a digital representation of all the physical and functional characteristics of a building through its entire life cycle (Isikdag and Zlatanova, 2009; Isikdag and Zlatanova, 2009; van Nederveen et al., 2014). The trouble, lack of indoor geometry and semantic information can be smoothed by employing IFC building model which contains detailed semantic and geometric information. Clear definition of building elements like storeys, door, windows, spaces in IFC model can provide pivotal information for topology derivation and detailed physical, functional characteristics contained in each element is enable intelligent routing and other indoor applications. Integrated spatial relationship between building elements in IFC such as a stair can link two floors together and wall can be a container of openings can also facilitate the derivation of the topology network. Semantically rich 3D models can provide critical information for navigation.

In this paper, we present a method which combined a straight media axis transformation algorithm (S-MAT) with IFC building model to reconstruct indoor geometric topology network. This derived topology network aimed at facilitating the decision making for different in-building navigation. The remainder of this paper is organized as follows. Next section depicts the research background about IFC Building Model and topology deviation methods. Section 3 elaborates on a multistep deviation process including semantic cleaning, walkable features extraction, Multi-Storey 2D Mapping and S-MAT implementation for generating topography information from existing indoor building model data given in IFC. Section 4 illustrates the applicability of the approach involving a typical IFC model. Sections 6 concludes by outlining the advantages of using the approach for topology derivations and overviews the future developments.

\section{RESEARCH BACKGROUND}

\subsection{IFC Building Model}

IFC is a standardized open data model developed for BIM developed by the International Alliance for Interoperability (IAI) 
(BuildingSMART, 2013), which is used for facilitating interoperability in the building industry and sharing information among participants (El-Mekawy et al., 2012). For the semantics information, IFC data models are semantics rich as they cover all physical and functional characteristics of the building. All of the spatial relationship between building elements are maintained in IFC with a hierarchical manner. Figure 1 represents the semantics relationship of IFC building element. For the geometry representation, IFC model can usually be created by multiple geometry including B-rep (Foley et al., 1994), Sweep volumes and CSG.

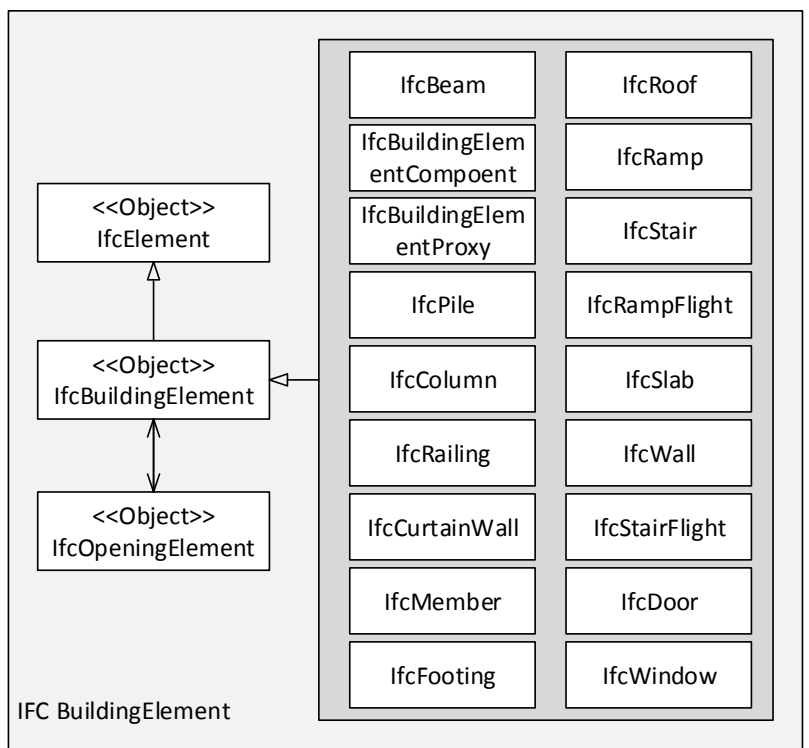

Figure 1. Semantics Relationship of IFC Building Element

\subsection{Topology Deviation Method}

Topology network explicitly represents the topological relationship among geographical features. These relationships includes connectivity, adjacency, inclusion and intersection (Egenhofer and Herring, 1990; Van Oosterom, 1993; Zlatanova et al., 2002). It is critical to explore a feasible way for building the connectivity relationships among 3D geographical features. Three kinds of method for topology network deviation (pure geometry- based, geometry semantics-based and pure semantics-based) are mainly used.

Pure Geometry-based: There is no semantics information involving in regeneration topology network in this method. (Lee, 2001) developed a topology data model which represents topological relationships, adjacency and connectivity relationships, between 3D spatial objects in built environments by using a straight MAT algorithm.

Geometry-Semantics-based: Most reaearches were based on both semantics and geometry information. $\mathrm{Li}$ and $\mathrm{He}$ (2008) and Li (2012) defined a 3D indoor navigation ontology for automatically conversion from a geometry model to a semanticbased topological network. Taneja and East (2011) used the straight MAT concept to transform IFC information into geometry networks, which need involve many manual operations. Chen and Huang (2014) present a method of constructing path networks directly from a revit building model. Two types of network construction algorithms, visibility graph and approximate MAT were presented and compared.which combined network analysis with Building Information Model (BIM) to facilitate the decision making for rescue operations

Pure Semantics-based: Some researchers used a door-to-door method to derive a visibility which is a network whose vertices are the vertices of obstacles and whose arcs connected all vertex pairs that are visible to each other. Based on the constructed graph, the shortest paths between initial points and destination can be found by using the shortest path algorithms, such as Dijkstra's algorithm (Dijkstra 1959). Lorenz et al. (2006) propose a hybrid spatial model for indoor environments. The model consists of hierarchically structured graphs with edges and nodes.

\section{TOPOLOGY DERIVATION FROM IFC MODEL}

\subsection{Semantics Cleaning}

Semantics cleaning aimed at filtering these elements out that have meaningful usage in topology derivation. There are about 900 classed defined in IFC schema and these classes are used store different information, such as relationship, attribute, geometry representation and element type etc. (de Laat and van Berlo, 2011). Many researches have already investigated information models that can be used for indoor navigation (Brown et al., 2013; Diehl et al., 2006). Based on these investigations, very few of these classes are relevant for topology derivation. Therefore, a semantic filtering method was used to screening these meaningful objects out. Figure 2 presents the workflow diagram for the semantics cleaning.

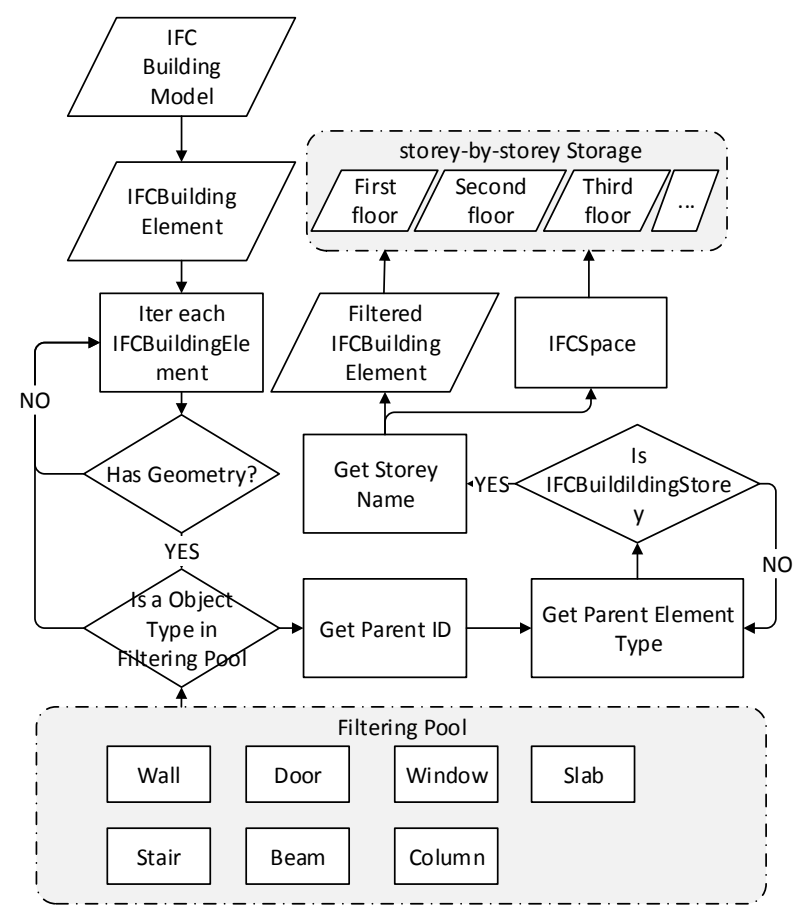

Figure 2. Workflow for the Semantics Cleaning

As shown in Figure 2, for each "IFCBuildingElement" existed in the IFC File, it should be checked whether it contains geometry. Those elements that do not have geometry would be ignored. Because very few of classes are relevant for topology derivation, a filtering pool consisted by seven types of the "IFCBuildingElement" is created in the filtering process. For topology derivation, each storey has different topology network, 
the element obtained from IFC file should be preserved storeyby-storey, so it is necessary to identified belonging indormation of each filtered elements. Most of the "IFCBuildingElement" like Wall, Stair, Slab, Beam and Column have a parent node with the type of "IFCBuildingStorey". However, door and window elements always beyond to an "IFCOpeningElement", which's parent node type is wall type. So at least two times upward iteration should be applied to door and window element for finding which storey they are belonging. Meanwhile, the relevant class, "IFCSpace" can be filtering out with the rid of storey information.

\subsection{Walkable Features Extraction}

After semantics filtering, elements are classified storey-bystorey. The inner structure for each storey is very complicated, there are many rooms, corridor, office and other features. In order to facilitate the derivation of the inner topology network, walkable features extraction related to the corridor elements is the most important. Abundant semantic information in IFC model can provides an effective method to automatic extract walkable features. Based on the filtered "IFCSpace" of each storey, it is used to provided all information about the space as a functional area or volume within a spatial structure. There are several attributions like "Name", "Description", "Long Name" and "ObjectType" in "IFCSpace", "ObjectType" can be used for identified the functional category of the space.

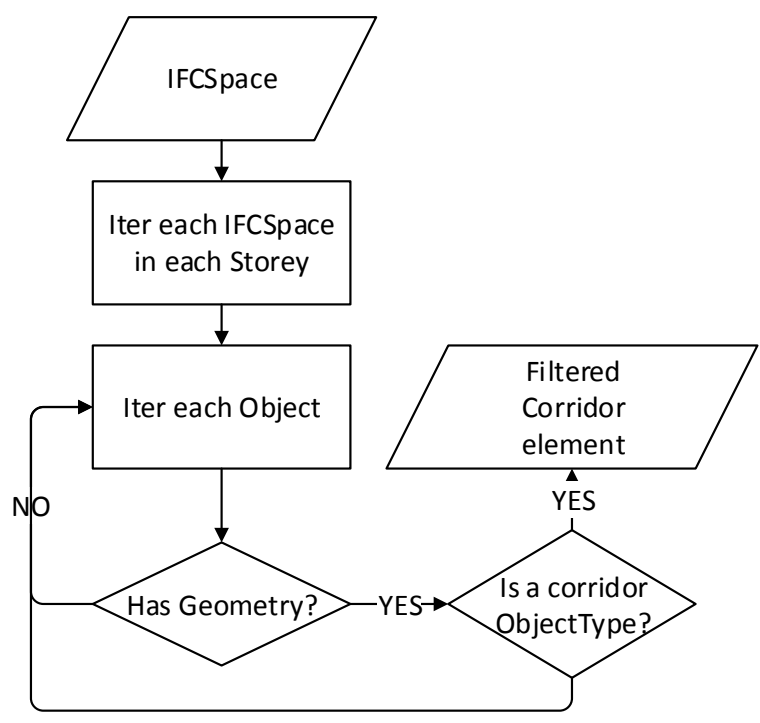

Figure 3. Workflow for walkable features extraction

Figure 3 presents the workflow diagram for walkable features extraction. For each "IFCSpace" in each storey, we should iterate all of the object and checked whether it contains geometry. Those elements that do not have geometry would be ignored. Then, when the obtained "ObjectType" is a "corridor" type, this object will be retained.

\subsection{Multi-Storey 2D Mapping}

Although 3D data models have been developed and implemented for geo-analyses and 3D visualization, they have some limitation with representing topological network because of the complex 3D geometric computational problems involved. In order to alleviate the problem, a combinatorial data model proposed by Lee and Kwan was employed to interpret the topological term among 3D object by drawing a dual graph in this paper. In order to facilitate S-MAT implementation, the object represented in three-dimensions of each storey should be mapping to the $2 \mathrm{D}$ planar for picking up the xy coordinates of nodes and maintain topological consistencies. Meanwhile, the semantic information existed in the original element would be retained in their respective edge and node. These mapping result can represent the basic topological relationships and connectivity relationships and is the base of topology network construction.

\subsection{Topology deviation from S-MAT}

The straight MAT algorithm developed by Lee (2004) can produce the skeleton graph of the 3D indoor environment, which based on a series of spatial points derived from a closed polygon. The S-MAT is generated by the angular bisectors of each pair of neighbouring edges. A segment of the angular bisector of these two neighbouring edges is added in the set of line segments of the medial axis. Those bisectors generated from all convex vertices of polygon $\mathrm{P}$ is called rays. When two neighbouring rays intersect a point and environ a thiessen polygon, the end type vertex would be assigned to the corresponding rays. Make rays which starts from the intersection point of the two parent rays and is a segment of the bisector of edge $e_{i}$ and $e_{i+2}$ or $e_{i-1}$ and $e_{i+1}$. After scanning all of the neighbouring bisector rays, a list of new rays is obtained. The process is ended when two rays of opposite direction remain.

After S-MAT implementation, the skeleton of the corridor can be obtained. An algorithm combining closest distance algorithm and door to door algorithm is employed to integrate door and stair to the prime network. For each door and stair, it should be checked whether it can connect to the skeleton of the corridor. Those elements that have no connection with the corridor should connect with its adjacent door or stair. At last, all of door and stair elements would be add into the derived topology for generating an integrated topology navigation network.

\section{CASE STUDY}

In this section, the proposed topology derivation method is tested with a two floor IFC building model named "091210Med_Dent_Clinic_Arch.ifc", which contains abundant geometry and semantics information. The elements in this model is sufficient for topology derivation.

4.1 First Step-semantics cleaning: the model before and after semantic cleaning were presented in Figure 4. This building is a two floor IFC building model, which contains abundant semantics information. The building model after semantic cleaning contains wall, door, window, stair, slab, beam and column objects.

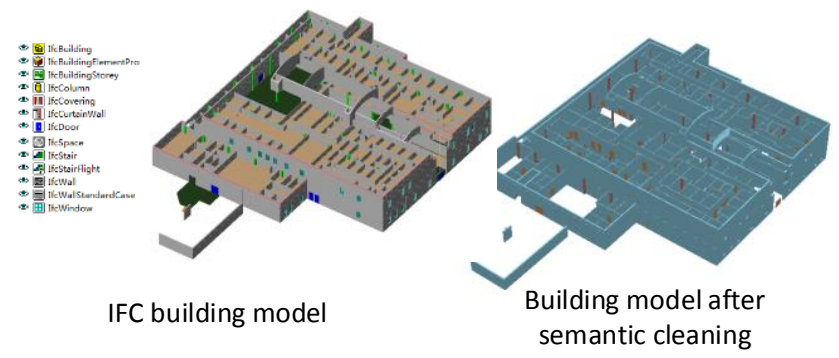

Figure 4. Original IFC model and model after semantic cleaning 
4.2 Second Step-walkable feature extraction: Based on the extracted "IFCSpace", walkable features can be obtained. Figure 5 presents the results of walkable features extraction. The above pictures are "IFCSpace" elements of two floor. The pictures drawing with gray are the respective corridor features. The blue parts are those element with special functions. They can be separated easily according to the "objecttype" attribution.
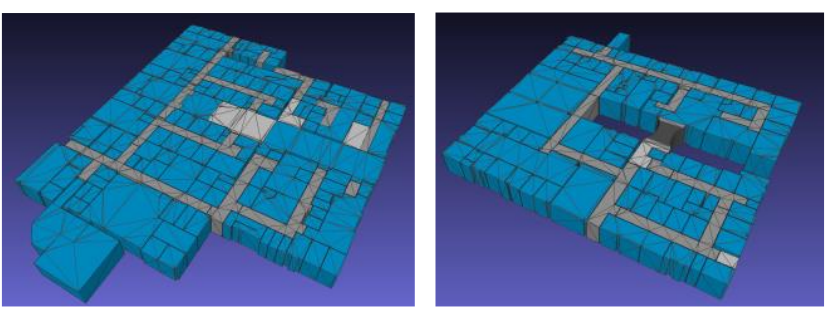

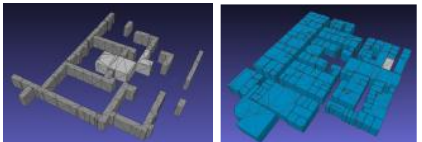

First Floor

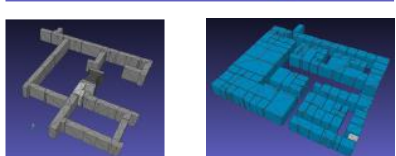

Second Floor
Figure 5. Results of walkable features extraction

4.3 Third Step-Multi-storey 2D Mapping: After Multi-storey 2D mapping, we can obtained the $\mathrm{x}, \mathrm{y}$ coordinates of the whole filtered object in each storey. The results of multi-storey 2D mapping were shown in Figure 6. In the figure, corridors are presented by green, doors are draw with blue, red lines represent stair elements and black lines are wall elements. Preparing for S-MAT implementation, 2D points of the walkable features polygon can be obtained. These points should organize by anticlockwise.

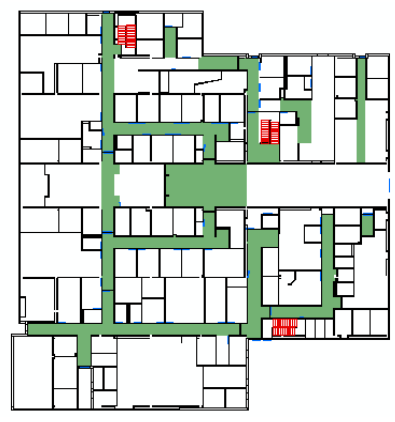

First Floor

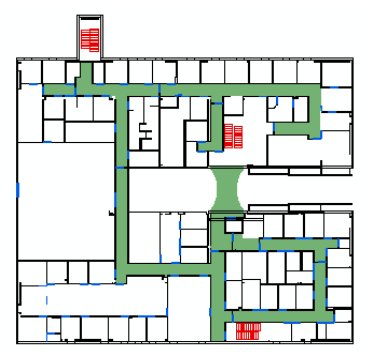

Second Floor
Figure 6. Results of Multi-storey 2D Mapping

4.4 Fourth Step-S-MAT implementation: As shown in Figure 7, the MAT network of each floor can be obtained after S-MAT implement, which use the walkable features as input. In above pictures in Figure 7, we can see the polygon of the walkable features have a hole. In the process of the S-MAT, the inner polygon should be wipe off and the order of the points derived from the polygons should be anticlockwise. After adding door and stair elements, the whole topology network are presented in below pictures in Figure 7. The topology network of each storey can be connected by stairs. This derived topology network can support multi-storey indoor navigation.
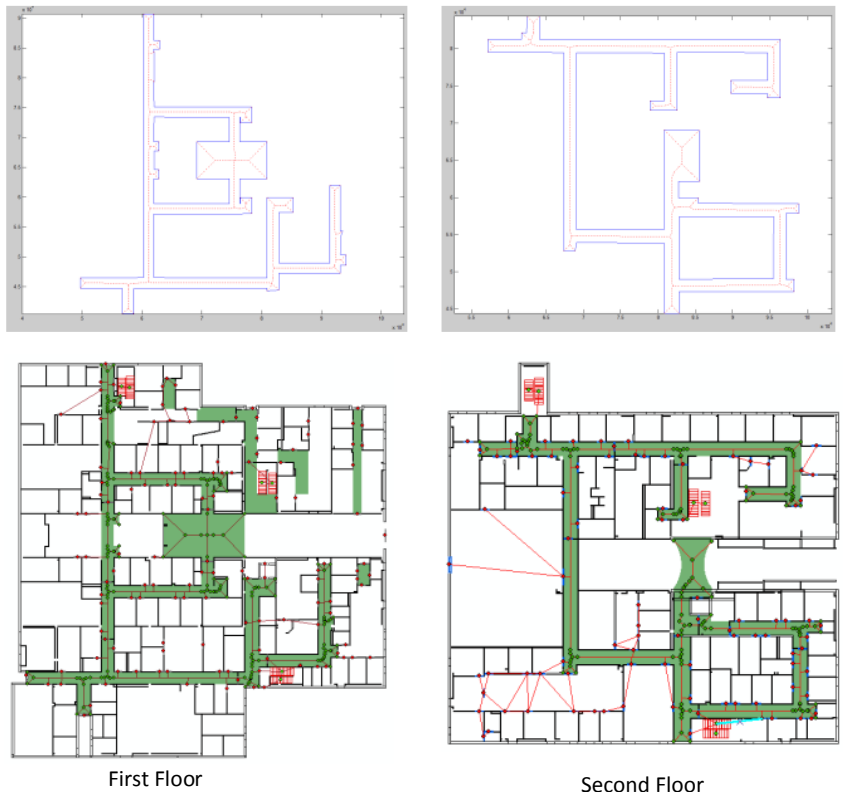

Second Floor

Figure 7. Result of S-MAT implementation

\section{CONCLUSION AND FUTURE WORK}

In this paper, the authors presented a multi-step deviation process including semantic cleaning, walkable features extraction, multi-Storey 2D Mapping and S-MAT implementation to automatically generate topography information from existing indoor building model data given in IFC. The abundant information inside the BIM models can facilitate the topology derivation greatly. The cases study shows the performance of this method, which is enable to generate a multi-storey topology network aiming at indoor navigation. However, as described in walkable feature extraction, the corridors are obtained from "IFCSpace" which defined in a perfect IFC model. In the cases of lack of IFCSpace, a new method should be designed for obtaining the walkable features. The main goal of our future work is to creatively integrate current powerful IFC information to enable more intelligent application such as indoor emergency evacuation and indoor facilitate management.

\section{ACKNOWLEDGEMENTS}

The work is Supported by the Open Fund of Key Laboratory of Urban Land Resources Monitoring and Simulation, Ministry of Land and Resources (Project No. KF-2015-01-027).

\section{REFERENCES}

Brown, G., Nagel, C., Zlatanova, S. and Kolbe, T.H., 2013. Modelling 3D topographic space against indoor navigation requirementsProgress and New Trends in 3D Geoinformation Sciences. Springer, pp. 1-22.

BuildingSMART, 2013.

Chen, A.Y. and Huang, T., BIM-Enabled Decision Making for In-Building Rescue Missions, Computing in Civil and Building Engineering (2014). ASCE, pp. 121128.

de Laat, R. and van Berlo, L., 2011. Integration of BIM and GIS: The development of the CityGML GeoBIM 
extensionAdvances in 3D geo-information sciences. Springer, pp. 211-225.

Diehl, S., Neuvel, J., Zlatanova, S. and Scholten, H., 2006. Investigation of user requirements in the emergency response sector: the Dutch case, Second Symposium on Gi4DM, pp. 25-26.

Egenhofer, M.J. and Herring, J., 1990. A mathematical framework for the definition of topological relationships, Fourth international symposium on spatial data handling. Zurich, Switzerland, pp. 803-813.

El-Mekawy, M., Östman, A. and Hijazi, I., 2012. An Evaluation of IFC-CityGML Unidirectional Conversion. International Journal of Advanced Computer Science \& Applications, 3(5).

Foley, J.D., Van Dam, A., Feiner, S.K., Hughes, J.F. and Phillips, R.L., 1994. Introduction to computer graphics, 55. Addison-Wesley Reading.

Isikdag, U. and Zlatanova, S., 2009. A SWOT analysis on the implementation of Building Information Models within the Geospatial Environment. Urban and Regional Data Management, CRC Press, The Netherlands: 15-30.

Isikdag, U. and Zlatanova, S., 2009. Towards defining a framework for automatic generation of buildings in CityGML using building Information Models3D geoinformation sciences. Springer, pp. 79-96.

Isikdag, U., Zlatanova, S. and Underwood, J., 2013. A BIM-Oriented Model for supporting indoor navigation requirements. Computers, Environment and Urban Systems, 41: 112-123.

Lee, J., 2001. 3D data model for representing topological relations of urban features, Proceedings of the 21st Annual ESRI International User Conference, San Diego, CA, USA.

Lee, J. and Kwan, M.P., 2005. A combinatorial data model for representing topological relations among 3D geographical features in micro - spatial environments.
International Journal of Geographical Information Science, 19(10): 1039-1056.

Li, Y., 2012. Building Information Model for 3D Indoor Navigation in Emergency Response. Advanced Materials Research, 368: 3837-3840.

Li, Y. and He, Z., 2008. 3D indoor navigation: a framework of combining BIM with 3D GIS, 44th ISOCARP congress.

Lorenz, B., Ohlbach, H.J. and Stoffel, E., 2006. A hybrid spatial model for representing indoor environmentsWeb and Wireless Geographical Information Systems. Springer, pp. 102-112.

Rueppel, U. and Stuebbe, K.M., 2008. BIM-based indoor-emergency-navigation-system for complex buildings. Tsinghua Science \& Technology, 13: 362-367. Shi, Y., Nakagawa, M. and Shibasaki, R., 2004. Reconstruction of "Next-generation" 3D Digital Road Model from Three Linear Scanner Images. Proceedings of ACRS, Thailand.

Taneja, S. and East, E.W., 2011. Transforming an IFCbased Building Layout Information into a Geometric Topology Network for Indoor Navigation Assistance. ASCE Reston, VA.

van Nederveen, S., Wolfert, R. and van de Ruitenbeek, M., 2014. From BIM to life cycle information management in infrastructure. eWork and eBusiness in Architecture, Engineering and Construction: ECPPM 2014: 115 .

Van Oosterom, P.J.M., 1993. Reactive data structures for geographic information systems. Oxford University Press New York.

Zlatanova, S., Rahman, A.A. and Pilouk, M., 2002. Trends in 3D GIS development. Journal of Geospatial Engineering, 4(2): 71-80. 